Tobias Böhmelt/Ulrich H. Pilster

\title{
Zur Problematik kollektiven Handelns
}

Eine quantitative Studie internationaler Umweltregime

\begin{abstract}
Zahlreiche Studien argumentieren, dass es in größeren Gruppen weniger wahrscheinlich ist, ein Kollektivgut optimal bereitzustellen. Der nachfolgende Aufsatz ordnet sich in diese Reihe von Forschungsarbeiten ein, indem die Bereitstellung von Umweltgütern durch internationale Regime untersucht wird. Verschiedene Kollektivhandlungsprobleme und deren Lösungen wurden seitens der Regimetheorie zwar bereits erforscht, jedoch fehlt das Wissen, auf welche genauen Merkmale der Bereitstellungssituation diese Handlungsprobleme zurückzuführen sind. Die folgende Arbeit disaggregiert daher zunächst diese Merkmale in nachfrageseitige Gütereigenschaften (d.h. öffentliche Güter, Externalitäten und Allmendegüter) und Gruppengröße, und untersucht anschließend empirisch deren Wirkung auf die Kooperationstiefe internationaler Umweltregime. Die Autoren zeigen, dass größere Regime Probleme haben, öffentliche Umweltgüter optimal zur Verfügung zu stellen. Dies trifft auch auf Externalitäten, jedoch nicht auf Allmendegüter zu.
\end{abstract}

\section{Einleitung ${ }^{1}$}

Unter welchen Umständen kooperieren Akteure innerhalb einer Gruppe, um ein gemeinsames Interesse effektiv bzw. optimal umzusetzen (Taylor 1987: 8)? Die bisherige Forschung bietet eine Reihe von Antworten auf diese Frage. Die wohl bekannteste Erklärung vertritt Mancur Olson in seinem Buch »The Logic of Collective Action« (1965). Olson argumentiert darin, dass je größer eine Gruppe ist, desto weniger ist sie in der Lage, ein gemeinsames Ziel zu erreichen (vgl. Oye 1985). Obwohl oft zitiert, zweifeln zahllose Studien an den Schlussfolgerungen, die Olson hinsichtlich des Effekts von Gruppengröße auf die Bereitstellung von kollektiven Gütern zieht. Todd Sandler (1992: 61-62) etwa schreibt, dass »we have seen a number of noteworthy exceptions to Olsonian propositions, such as the exploitation of the large by the small or the inverse relationship between collective provision and group size«. Michael Taylor (1987: 12) argumentiert weiter, dass »Olson's model [...] is rather unrealistic. Accordingly, not too much value should be attached to conclusions derived from it, including conclusions about the effects of increases in group size«. Und Russell Hardin (1982) kritisiert, dass »the critical value may not be the size of the group as a whole but the size of the smallest subgroup able to share costs in a way that allows all its own members to benefit«.

1 Die Autoren danken Thomas Gehring, Sebastian Krapohl, Benjamin Faude und den anonymen Gutachterinnen und Gutachtern der Zeitschrift für Internationale Beziehungen für die zahlreichen Anmerkungen und Verbesserungsvörschläge. 
Mit anderen Worten ist Olsons »Gruppengrößenprinzip« aufgrund seiner Plausibilität in politikwissenschaftlichen Arbeiten zwar weit verbreitet, aber zugleich auch einer Vielzahl von Kritiken ausgesetzt, und sobald es mit empirischen Daten konfrontiert wird, verliert Olsons Modell oft an Glaubwürdigkeit. ${ }^{2}$ Zum Beispiel untersucht Scott Barrett $(2007 ; 1999)$ etwa die Bereitstellung von globalen öffentlichen Gütern, aber argumentiert - anders als Olson - dass die Wahrscheinlichkeit der Bereitstellung von verschiedenen Güterfaktoren abhängt. Barrett (2007) unterscheidet hier zwischen best-shot-Gütern, weakest-link-Arten oder aggregierten Bereitstellungsbemühungen von Akteuren und schlussfolgert, dass »global public goods are not all alike, and the differences that distinguish one type from another create constraining incentives for provision« (Barrett 2007: 9). Mit anderen Worten muss die Bereitstellung von öffentlichen Gütern nicht zwangsweise scheitern - es hängt vielmehr von den speziellen Charakteristika eines solchen Gutes ab (Barrett 2007: 20; vgl. Harris 2007; Sandler 2004). Katharina Holzinger (2001; Runge 1984) untersucht spieltheoretisch, wie bestimmte Charakteristika kollektiver Güter (Nichtausschließbarkeit und das Rivalitätsprinzip) und die sozialen Situationen, in denen diese Güter bereitgestellt werden sollen, den Erfolg der Bereitstellung beeinflussen. Abschließend zeigen Mark Isaac et al. (1994), dass große Gruppen durchaus in der Lage sind, öffentliche Güter zur Verfügung zu stellen. Die Autoren stützen sich auf ein experimentelles Forschungsdesign und finden Hinweise dafür, dass Gruppen von 40 oder 100 Akteuren ein kollektives Gut effizienter bereitstellen als Gruppen mit weniger Mitgliedern.

Dieser kurze Überblick einiger empirischen Arbeiten zu Problemen kollektiven Handelns betont zum einen nicht nur die Kritk an Olson, sondern auch, dass das Gruppengrößenprinzip empirisch tatsächlich noch umstritten ist und es keinen Konsens darüber gibt, wie Kollektivgüter effektiv bereitgestellt werden können (z.B. Harris 2007; Isaac et al. 1994: 2). Weiterhin gehen diese Arbeiten über Olsons Grundmodell hinaus, indem neben den nachfrage- auch die produktionsseitigen Eigenschaften der Güter selbst, Akteurs- und Gruppenmerkmale, sowie Bedingungen der rechtlichen, institutionellen und sozialen Umwelt, unter denen Güter bereitgestellt werden, untersucht werden: Je nachdem, welche dieser Merkmale vorliegen, ergeben sich verschiedene Typen strategischer Konstellationen und damit auch ganz unterschiedliche Prognosen für die Bereitstellung kollektiver Güter (Holzinger 2001: 134; Runge 1984; Sandler 2004).

Die folgende Arbeit ordnet sich in diese Reihe von Forschungsarbeiten ein, indem die Bereitstellung von Umweltgütern durch internationale Regime, d.h. »Prinzipien, Normen, Regeln und Entscheidungsverfahren, um die die Erwartungshaltungen der Akteure konvergieren« (Krasner 1983: 2; Simmons/Martin 2002: 193), untersucht

2 Vgl. z.B. Frohlich/Oppenheimer (1970: 108); Chamberlain (1974); Hardin (1970); Keohane/ Ostrom (1995); Sandler (2004); Schelling (1973); Shimizu/Sandler (2002); Taylor (1987: 12). Für einen umfangreiche Sammlung von Forschungsarbeiten, die die Theorie der (globalen) öffentlichen Güter über ihre ursprüngliche Fundierung in der normativen neoklassischen Wohlfahrtsökonomie hinaus als positive Theorie nutzen, um reales Akteursverhalten bei der Bereitstellung (globaler) öffentlicher Güter zu erklären, vgl. Kaul et al. (2003). 
wird. Verschiedene Kollektivhandlungsprobleme und deren Lösungen wurden seitens der Regimetheorie zwar umfangreich erforscht, ${ }^{3}$ jedoch fehlt hier das Wissen, auf welche genauen Merkmale der Bereitstellungssituation diese Handlungsprobleme zurückzuführen sind (vgl. Harris 2007: 220). So exisitert im Bereich der Regimeforschung auch keine quantitative Studie, die diese Fragestellung systematisch untersucht. Poteete/Ostrom (2008) argumentieren, dass das Fehlen eines hinreichend großen Datensatzes ausschlaggebend für die bisherige geringe Anzahl an quantitativen Arbeiten innerhalb der Regimeliteratur ist.

Wir tragen mit diesem Aufsatz zu der bisherigen Forschung bei, indem wir diese Merkmale von Bereitstellungssituationen zunächst in nachfrageseitige Gütereigenschaften - d.h. öffentliche Güter, Externalitäten und Allmendegüter ${ }^{4}$ - und Gruppengröße disaggregieren und anschließend deren Wirkung auf die Kooperationstiefe internationaler Umweltregime empirisch untersuchen. Dabei sind unsere zentralen Argumente wie folgt: Erstens sind größere Gruppen weniger in der Lage, öffentliche Umweltgüter optimal bereitzustellen oder Externalitäten effektiv zu beseitigen. Dies liegt insbesondere daran, dass sie sich durch höhere Organisations- bzw. Transaktionskosten auszeichnen (vgl. Dixit/Olson 2000; Mueller 2003: 35; Oye 1985: 19; Taylor 1987: 105). Zweitens argumentieren wir, dass größere Gruppen eher einen optimalen Schutz von Allmendegütern sicherstellen können, da sich hier die Akteuere mit steigender Gruppengröße in Untergruppen formieren werden, die starke Anreize daran haben, ihren Langzeitnutzen zu wahren und so letztendlich kooperieren (Snidal 1991a; 1991b; Keohane/Ostrom 1995: 6). Unsere Ergebnisse betonen, dass die Unterscheidung zwischen verschiedenen Gütertypen essentiell für das Verständnis von Problemen kollektiven Handelns im Rahmen der Regimeforschung ist (vgl. Harris 2007: 221). Es zeigt sich, dass größere Regime in der Tat Probleme haben, öffentliche Umweltgüter optimal bereitzustellen. Die empirischen Analysen zeigen aber auch, dass diese negative Beziehung ebenfalls auf Externalitäten zutrifft, jedoch nicht auf Allmendegüter. Vielmehr scheint es, dass größere Gruppen wahrscheinlich besser in der Lage sind, Allmendegüter zu schützen.

Der wissenschaftliche Beitrag unserer Studie ist im Wesentlichen in der empirischquantitativen Untersuchung von Merkmalen der Bereitstellungssituationen von Problemen kollektiven Handelns im Rahmen der Regimeforschung gegeben. Darüber

3 Vgl. Harris (2007); Hasenclever et al. (1997); Ostrom (1990); Poteete/Ostrom (2008); Zangl (2003); Zürn (1992; 1998).

4 Bei kollektiven oder öffentlichen Gütern können individuelle Akteure nicht von dem Konsum ausgeschlossen werden (Nichtausschließbarkeitsprinzip) und die konsumierte Menge eines bestimmten Akteures beeinträchtigt nicht die verfügbare Menge des Gutes für andere Staaten (Nichtrivalitätsprinzip). Für Allmendegüter oder common pool-Ressourcen trifft das Nichtausschließbarkeitsprinzip ebenfalls zu, aber der Konsum dieses Gutes durch einen Akteur verringert die verfügbare Menge für die anderen Staaten innerhalb der Gruppe (Rivalitätsprinzip) (Barkin/Shambaugh 1996; Hardin 1970; Ostrom 2003). Externalitäten werden in der Regel als »Spezialfall« von öffentlichen Gütern behandelt. Aufgrund der Definition als »problems where one country is the victim of another country's outsourcing of a certain problem, with this relationship not being symmetrically « (Breitmeier et al. 1996: 25; Mueller 2003: 25; Ringquist/Kostadinova 2005: 94) analysieren wir diesen Gütertypus aber getrennt von öffentlichen Gütern (vgl. Schelling 1973). 
hinaus haben unsere Ergebnisse aber auch politische Implikationen für die gegenwärtigen Debatten hinsichtlich der Neuverhandlung von internationalen Verträgen und insbesondere internationalen Umweltregimen (vgl. z.B. Najam 2003; Victor 2006: 95). Zum Beispiel wurde versucht im Rahmen der Post-Kyoto-Verhandlungen in Kopenhagen 2009 zu entscheiden, ob und welche zusätzlichen Akteure innerhalb eines neuen Regelwerkes vertraglich gebunden werden sollen, um das Klimaschutzproblem zu lösen. In einer ähnlichen Position befindet sich das europäische Regime zur Verhinderung weitreichender Luftverschmutzung (long-range transboundary air pollution, LRTAP), welches in der Vergangenheit wiederholt die eigenen Protokolle änderte, um Ziele oder die Mitgliedschaft einzelner Staaten zu regeln (Perrin/Bernauer 2010). Durch den gegenwärtigen Schwerpunkt des LRTAP auf Externalitätsproblemen durch Luftverschmutzung, entsteht die Frage, was sich ändern könnte, wenn zusätzliche Staaten in das Vertragswerk integriert werden.

Vor diesem Hintergrund wird im nächsten Abschnitt zunächst der Forschungsstand zu internationalen Umweltregimen kurz wiedergegeben, um das für unsere Arbeit notwendige Hintergrundwissen zu bilden. Im darauffolgenden Abschnitt erläutern wir detailiert die Beziehung der Gütertypen zu dem Erfolg kollektiven Handelns, unter dem Einfluss der Größe von Akteursgruppen. Dies macht es uns möglich, eine Reihe von Hypothesen aufzustellen, die wir im Anschluss anhand einer quantitativen Analyse testen. Die Daten dafür sind der International Regimes Database (IRD) (Breitmeier et al. 1996; 2006; Young/Zürn 2006) entnommen. Der letzte Abschnitt fasst die Ergebnisse zusammen und diskutiert deren Implikationen.

\section{Die Forschungsstand internationaler Regime - ein kurzer Überblick}

Ein Großteil der Studien der Regimeliteratur konzentriert sich auf den Bereich der »Regimeeffektivität«, d.h. inwiefern ein Regime in der Lage ist, ein bestimmtes Problem zu lösen und welche Faktoren diese Problemlösungsfähigkeit beeinflussen. Die bestehende Literatur fasst die Einflussvariablen von Regimeeffektivität in der Regel unter den Faktoren »Problemstruktur« und »Regimedesign« zusammen, wobei der Einfluss von Regimedesign auch von der Struktur eines Regimeproblems abhängig ist (vgl. Underdal 2002; Hovi et al. 2003).

In Bezug auf das Design internationaler Regime identifizieren Edward Miles et al. (2002) etwa den institutionellen Hintergrund, die Verteilung staatlicher Macht als auch bestimmte Fähigkeiten und Eigenschaften wie Führungsqualität oder den Einfluss von epistemic communities als wichtige Determinanten (vgl. Koremenos et al. 2001). Ronald Mitchell (1996; 2002: 18; 2003: 451) konzentriert sich darüber hinaus auf die Unterscheidung zwischen dem primären Regelwerk einer Institution, d.h. den Anforderungen hinsichtlich staatlicher Verhaltensänderung, Überwachungs- und Informationssystemen und den institutionellen Regeln im Falle von Vertrags- und Regelverletzungen.

In Bezug auf das zugrundeliegende Problem eines Regimes hat der problemstrukturelle Ansatz (vgl. Zürn 1992) ursprünglich eine Erfassung von Regimeproblemen 
anhand von spieltheoretischen Konzepten wie 2x2-Koordinations-, Kollaborationsund Versicherungsspielen versucht (vgl. Hasenclever et al. 1997; Martin 1992; Martin 1995; Mitchell 2006: 80). Ein zweiter Ansatz ist in der Unterscheidung zwischen Wertkonflikten, absolut und relativ bewerteten Gütern auszumachen (Rittberger/Zürn 1990). Weiss/Jacobson (1998: 536) identifizieren hier vier Faktoren, wie etwa die Anzahl relevanter Akteure oder den Einfluss ökonomischer Anreize; weiterhin zwölf länderspezifische Variablen, z.B. Ländergröße und die politische Führung; zuletzt sechs Faktoren, die dem internationalen System zuzuordnen sind, z.B. den Einfluss anderer institutioneller Vereinbarungen oder die öffentliche Meinung. Young (1999) findet auch Hinweise für den Einfluss von Regimedesign auf das Verhalten von Akteuren, berücksichtigt aber die Problemstruktur nur als untergeordnete Variable. Abschließend verwenden Miles et al. (2002: 37) Inkongruenz, Asymmetrie und die sogenannte kumulierte cleveage-Struktur, um das Ausmaß an »Malignität« zu bestimmen. Dieses wird im Anschluss mit der Unsicherheit von Akteuren hinsichltich der Konsequenzen ihres Handelns verbunden, um ein bestimmtes Umweltproblem zu erfassen (vgl. Zürn 1992). Die Autoren finden, dass größere Unsicherheit bei einem Problem und größere Malignität einen negativen Einfluss auf Regimeeffektivität haben.

Vor diesem Hintergrund stellt die Effektivität eines Regimes im Wesentlichen den kooperativen Erfolg von Akteuren unter dem Dach einer organisierten Gruppe dar. Nichtsdestoweniger weist die Literatur eine Vielzahl an Vorschlägen auf, wie Regimeeffektivität nun zu verstehen sei und so ist es schwierig, eine Konsensdefinition dafür zu finden (Ward 2006: 154). Auf der einen Seite existieren Versuche einer Definition des eigentlichen Gegenstandes, der eine Evaluationsgrundlage (d.h. »baseline«) bilden soll. Die Frage ist dabei insbesondere, ob der Fokus nun auf Veränderungen im Sinne eines »Vorher-Nacher-Vergleichs« im umweltschädlichen Verhalten des Menschen oder der Umweltqualität an sich liegen soll (Bernauer 1995: 355; Mitchell 2002: 17; Ward 2006: 154).

In diesem Zusammenhang schlagen Studien (vgl. z.B. Miles et al. 2002) nun (1) den »output« eines Regimes im Sinne von produzierten Regeln und Verhaltensvorschriften als Massstab von Effektivität vor; (2) die relative Verbesserung des menschlichen Umweltverhaltens und -bewusstseins im Vergleich zu einem kontrafaktuellen Zustand vor, in dem das fragliche Regime eben nicht existieren würde (»outcomeEbene«); und (3) die Zielerreichung eines kollektiven Optimums für das Umweltproblem in seiner Gesamtheit vor (d.h. inwieweit ein angestrebter Umweltzustand durch das Regime erreicht werden konnte; »impact-Ebene «). ${ }^{5}$

Zusammenfassend lässt sich für unsere Fragestellung - wie in der Einleitung betont - anmerken, dass die verschiedenen Kollektivhandlungsprobleme und deren Lösungen seitens der Regimetheorie zwar umfangreich erforscht wurden, es jedoch unklar ist, auf welche genauen Merkmale der Bereitstellungssituation diese Handlungs-

5 Eine Synthese der Vorschläge (2) und (3) wird in der Literatur als »Oslo-Potsdam-Lösung« (Helm/Sprinz 2000) bezeichnet. 
probleme zurückzuführen sind. ${ }^{6}$ Wir versuchen diese Forschungslücke zu schließen. Umweltprobleme auf regionaler oder internationaler Ebene sind in der Regel auf das Versagen kollektiven Handelns zurückzuführen, was zu einer suboptimalen »Bereitstellung« von Umweltqualität führt (Young 1982: 282; Taylor 1987: 19; Ward et al. 2004: 151). Um dieses aktuelle oder zukünftige Problem kollektiven Handelns zu bewältigen, müssen Staaten kooperieren, d.h. es müssen gemeinsame Anstrengungen unternommen worden, um eigenes Verhalten und das anderer Akteure neu auszurichten und anzupassen. Um Kooperation zu erreichen, verlassen sich Staaten verstärkt auf die Möglichkeit, ein internationales Regime zu errichten, dass das Bereitstellen des Kollektivgutes Umweltqualität begünstigen soll (Krasner 1983: 2; Simmons/Martin 2002: 193).

Im Folgenden sind wir insbesondere daran interessiert, wie Regime, die eine Vielzahl von Akteuren vereinen, Umweltprobleme bewältigen können. ${ }^{7}$ Wir stützen uns dazu auf die umfassende Literatur zu Marktversagen (vgl. Agrawal/Goyal 2003: 63; Mueller 2003: 9; Ostrom 1990; 2003: 240; Sandler 2004: 45), indem wir die kollektiven Anstrengungen von Staaten als öffentliches Umweltgut, als das Abwenden einer Externalität oder als den Schutz eines Allmendegutes konzeptualisieren. Im Anschluss daran evaluieren wir den Einfluss von Gruppengröße auf Kooperationserfolg, d.h. Regimeeffektivität, konditional abhängig von jedem dieser drei Güterarten.

\section{Theorie: Der Einfluss von Gruppengröße auf die Bereitstellung kollektiver Umweltgüter}

\subsection{Gruppengröße und öffentliche Umweltgüter}

Olson (1965) zufolge können sich latente, d.h. große Gruppen nicht mit einem öffentlichen Gut versorgen, weil es aus Sicht eines einzelnen Akteurs rational erscheint, die Position des Trittbrettfahrers einzunehmen. Trittbrettfahren oder »free-riding« geschieht, da der Beitrag eines Einzelnen keine spürbare Wirkung erzielt und dieser Akteur daher umsonst in den Konsumgenuss eines öffentlichen Guts kommt, sein

6 Vgl. Harris (2007); Hasenclever et al. (1997); Ostrom (1990); Poteete/Ostrom (2008); Zangl (2003); Zürn (1992; 1998). Von einem analytischen Standpunkt aus lässt sich auch feststellen, dass alle bisherigen Studien ausnahmslos den Einfluss von Problemstruktur auf die Effektivität von Regimen direkt neben Regimedesign untersucht haben. Dies erscheint jedoch problematisch, wenn man bedenkt, dass institutionelles Design vielmehr eine intervenierende Variable zwischen Problemstruktur und Effektivität ist. Wir diskutieren diese methodische Herausforderung im Abschnitt unseres Forschungsdesigns.

7 In diesem Zusammenhang weisen wir auch auf das verwandte Argument hin, dass die Wahrscheinlichkeit der Bildung eines Regimes mit steigender Gruppengröße abnimmt (Oye 1985: 18). Im Folgenden konzentrieren wir uns jedoch auf die Bereitstellung kollektiver Güter nach der Bildung einer solchen Institution. 
Trittbrettfahren in einer großen Gruppe weniger auffällt und daher nicht sanktioniert wird. ${ }^{8}$

Vor diesem Hintergrund proklamiert das zentrale Argument, welches Gruppengröße mit dem Erfolg kollektiven Handelns verbindet, dass, je größer die Anzahl der Akteure einer Gruppe ist, desto größer sind die Kosten, um diese Gruppe effektiv zu organisieren. Aufgrund dieser hohen Organisationskosten ist es anschließend weniger wahrscheinlich, dass öffentliche Güter optimal bereitgestellt werden (Chamberlain 1974; Olson 1965: 46; Snidal 1996; Taylor 1987: 105). ${ }^{9}$

Organisationskosten manifestieren sich in der Regel durch einen zentralen Mechanismus: Der Vollzug institutioneller Regeln wird mit einer größeren Gruppe schwieriger, da a) Instrumente der Überwachung von staatlichem Verhalten und b) etwaige Sanktionierungsinstrumente teuer werden. ${ }^{10}$ Aufgrund des Nichtausschließbarkeitsprinzips öffentlicher Umweltgüter sind Instrumente der Überwachung staatlichen Verhaltens und Sanktionierungsinstrumente jedoch essentiell um free-rider-Verhalten auf Kosten von Kooperationsanstrengungen anderer Staaten zu unterbinden (Fearon 1998: 270; Hasenclever et al. 1997: 54; Koremenos et al. 2001: 773; Zangl 2003).

Überwachungssysteme stellen sicher, dass die Beiträge von Gruppenakteuren, die kooperieren möchten, auch sichtbar werden. Dies ist sehr bedeutend für die differenzierte Verteilung von selektiven Anreizen für die Mitglieder einer Gruppe, da die Wahrnehmung individueller Beiträge die Möglichkeit eröffnet, dass die Mitglieder

8 Es sei hier anzumerken, dass auch in priviligierten, d.h. kleinen Gruppen für Olson (1965) eine Tendenz zur suboptimalen Versorgung mit öffentlichen Gütern besteht. Allerdings sind solche Gruppen durch stärkere Interdependenzbeziehungen gekennzeichnet, so dass ein fehlender Beitrag eines Einzelnen eher mit Reputationsverlusten einhergeht. In kleinen Gruppen produziert der Einzelne zudem einen (relativ betrachtet) größeren Anteil des Gesamtnutzens als in großen Gruppen, was wiederum bedeutet, dass (unter der Annahme einer Gleichverteilung der Nutzen) der Grenznutzen höher ist als in großen Gruppen. Ein Einzelner wird somit weniger wahrscheinlich eine private Konsummöglichkeit finden, die einen noch höheren Grenznutzen erzeugt als sein Beitrag zum Kollektivgut. Wir danken einem anonymen Gutachter für diesen Hinweis.

9 Ein anonymer Gutachter deutete in diesem Zusammenhang richtigerweise auf die Bedeutung von k-Gruppen (Hardin 1982; Schelling 1973) hin. Diesem Argument zufolge ist nicht die nominale, sondern die k-Gruppengröße entscheidend für die Wahrscheinlichkeit der Güterbereitstellung (vgl. Snidal 1985): Die Bereitstellung eines öffentlichen Guts hängt somit weniger von der Gesamtgröße der Gruppe als von der Mindestzahl an Gruppenmitgliedern ab, die von der Kooperation untereinander selbst dann noch profitieren, wenn alle anderen Gruppenmitglieder als Trittbrettfahrer auftreten. Wenn diese k-Gruppen relativ klein ist, könnten theoretisch sogar nominal große Gruppen mit einem reinen öffentlichen Gut versorgt werden. Je kleiner diese k-Gruppe ist, desto weniger werden ihre Mitglieder aufgrund der damit verbundenen Interdependenzdichte durch Trittbrettfahren aus der Kooperation ausscheren. Wir stimmen im Wesentlichen dieser Argumentation zu, möchten aber gleichzeitig auf Folgendes hinweisen: Zum einen möchten wir unsere theoretischen Überlegungen hier auf dem Originalmodell Olsons (1965; vgl. Olson/Zeckhauser 1966) aufbauen und dieses im Anschluss einem empirischen Test im Rahmen internationaler Umweltregime unterziehen. Dies erlaubt uns nicht nur die Validität Olsons in diesem Forschungsfeld festzustellen, sondern auch unsere Argumentation an den einfach zu bestimmenden Charakteristika von öffentlichen Gütern aufzubauen. Zum anderen gehen wir im Abschnitt der Kontrollvariablen direkt auf das k-Gruppenargument ein, indem wir ein item berücksictigen, welches genau dies misst.

10 Fearon (1998: 270); Hasenclever et al. (1997: 54); Koremenos et al. (2001: 773); Sandler (2004: 32). 
der Gruppe strategisch interagieren können (Olson 1965: 43). Im Umkehrschluss heißt dies, dass nur die Möglichkeit, fehlende Beiträge, d.h. Defektieren von Staaten, auszumachen, effektives Sanktionieren bedingt (Axelrod/Keohane 1985: 235; Hasenclever et al. 1997: 188; North 1990: 57; Olson 1965: 45). Um das Argument hinsichtlich von Überwachungssystemen abzurunden: Je größer eine bestimmte Gruppe ist, desto teurer werden diese Instrumente, die die Beiträge von Staaten aufdecken und sichtbar machen. Aufgrund steigender Kosten wird unwahrscheinlich, dass eine solche Gruppe in ihren Anstrengungen, ein öffentliches Umweltgut bereitzustellen, auch erfolgreich ist.

Neben Überwachungssystemen sind Sanktionierungsmechanismen, wie z.B. das Zurückziehen von positiven, selektiven Anreizen oder »Bestrafungen«, notwendige Bedingungen, um die Akteure einer Gruppe zu Kooperation zu bewegen - besonders in Umgebungen, in denen das Defektieren offensichtlichen Nutzen bieten kann (vgl. Downs et al. 1996: 397; Kydd/Snidal 1993: 117). Dies trifft im speziellen auf öffentliche Umweltgüter aufgrund des Nichtausschließbarkeitsprinzips zu. Falls ein Regime keine derartigen Instrumente aufweist, gibt es keinen Grund, a priori anzunehmen, dass kollektive Umweltgüter in einer Gruppe unabhängig von ihrer Größe bereitgestellt werden (Frohlich/Oppenheimer 1970: 119). Auch Olson (1965: 36) argumentiert in dieser Richtung: »unless there is coercion or some other special device to make individuals act in their common interest, rational, self-interested individuals will not act to achieve group interests «. Wendet man nun denselben logischen Gedankengang wie zuvor an, so werden nicht nur Sanktionierungsmechanismen teurer, sondern auch das Bereitstellen eines öffentlichen Gutes wird umso weniger wahrscheinlich, je größer die Anzahl an Akteuren in einer Gruppe ist. In sehr großen Gruppen, oder wie in unserem Fall Regimen mit einer großen Zahl an Mitgliedern, wird es zunehmend schwierig, Sanktionen gegen einen bestimmten Staat zu verhängen und es ist wahrscheinlich, dass es Akteure in dieser Gruppe gibt, denen der Anreiz fehlt, solche Staaten zu bestrafen (Axelrod/Keohane 1985: 234; North 1990: 57). Zusammenfassend lässt sich nunmehr folgende Hypothese aufstellen:

H1: Je größer eine Gruppe ist, die ein öffentliches Umweltgut bereitstellen will, desto geringer ist die Wahrscheinlichkeit, dass ihr dies erfolgreich gelingt.

\subsection{Gruppengröße und Externalitäten}

Zur Veranschaulichung von Externalitäten mag uns das Beispiel Großbritanniens in den 1950ern und 1960ern dienen. Zu dieser Zeit wurden die Schornsteine von Fabriken, Häusern, etc. höher gebaut, um die starke Luftverschmutzung zu bekämpfen. Dies hatte zur Folge, dass die Verunreinigungen in die Atmosphäre gelangten und aufgrund des Regenwassers dort versäuerten. Im Anschluss trugen Winde diesen versäuerten Niederschlag nach Skandinavien, wo erhebliche negative Einwirkungen auf die Umwelt entstanden. Mit anderen Worten: Der Nutzen, das Gut »Umwelt « in Staaten wie Dänemark oder Norwegen zu genießen, sank, während der Nutzen des Verursacherstaates Großbritannien in keinster Weise negativ beeinflusst wurde. 
Wie aufgezeigt, konnten die skandinavischen Länder, hier das »Opfer«, nicht von den spill-over-Effekten, die durch Großbritannien verursacht wurden, ausgeschlossen werden. Dennis Mueller (2003: 27) argumentiert, dass diese Nichtausschließbarkeit letztendlich dazu führt, dass die exakt gleiche »Pareto-Optimalitätsbedingung« wie bei öffentlichen Umweltgütern gegeben ist. Im Gegensatz zu öffentlichen Gütern konsumieren die Akteure jedoch nicht dasselbe Gut (Schelling 1973: 385). Auch haben Externalitäten keinen Preismechanismus, um individuelle Aktionen zu koordinieren. Dies führt schließlich zu pareto-suboptimalen Ergebnissen (Mueller 2003: 25).

Demzufolge erscheint es logisch, dass teure Koordinationsmechanismen notwendig sind, um pareto-optimale Lösungen zu finden. Andererseits argumentiert Coase (1960), dass Akteure, die durch Externalitäten in ihrem Nutzen beeinträchtigt werden, dies effektiv durch bargaining beheben können. So könnten betroffene Staaten dazu befähigt sein, den Akteur, der die Externalität verursacht, zu kompensieren, damit dieser sein Handeln unterlässt, während man trotz dieser side-payments ein besseres Ergebnis als im status quo erzielt. Der Verursacherstaat würde demnach sein Handeln unterlassen, erhielte er eine Zahlung, die dem Nutzen der ursprünglichen Situation mit der Externalität gleicht. Der betroffene Staat würde für diese Zahlung aber weniger aufbringen müssen als den Verlust an Nutzen, der durch die fortdauernde Externalität verursacht ist. Nichtsdestoweniger nimmt Coase an, dass Transaktionskosten hier vernachlässigt werden können. Wir stimmen zu, dass Regime ihren Mitgliedern durch das signifikante Senken von Transaktionskosten helfen, Langzeitinteressen zu verfolgen und pareto-inferiore Situationen zu bewältigen (Hawkins et al. 2006: 15; Keohane 1984; Snidal 1996; Zangl 2003: 125). Dennoch wird eine nicht zu unterschätzende Kostenmenge auch trotz eines Regimes fortbestehen. Auch beweisen Dixit/Olson (2000; vgl. Mueller 2003: 35), unabhängig von der Annahme, ob Transaktionskosten nun vernachlässigt werden können oder nicht, dass Coases Lehrsatz zunehmend unplausibel wird, je größer eine Gruppe ist: Übereinkünfte zwischen den Akteuren drohen gebrochen zu werden und es besteht ein größerer Zeitaufwand, eine Lösung, mit der bestimmte Akteure Zahlungen an andere leisten, auszuhandeln. Dies erhöht zwangsläufig die Transaktionskosten, was es im Umkehrschluss weniger wahrscheinlich macht, Probleme kollektiven Handelns aufgrund von Externalitäten lösen zu können:

H2: Je größer eine Gruppe ist, die sich mit einer Externalität befasst, desto geringer ist die Wahrscheinlichkeit, dass ihr dies erfolgreich gelingt.

\subsection{Gruppengröße und Allmendegüter}

Das Regime zur Regelung des Fischfangs im Südpazifik (South Pacific Fisheries Forum Agency) ist ein Beispiel für den Schutz eines Allmendegutes, da die Menge an Fisch in dieser Region weniger wird, je mehr Anrainerstaaten im Fischfang involviert sind. Demzufolge erscheint es a priori plausibel, dass es umso unwahrscheinlicher wird, den Fischfang eines einzelnen Staates effektiv zu kontrollieren, je mehr 
Anrainerstaaten existieren. Dies hat zur Folge, dass es zum einen schwierig ist, einzelne Staaten direkt zu identifzieren, wenn bestimmte Fangquoten überschritten werden und dass es zum anderen nützlich erscheint, neben den Kooperationsbemühungen anderer Staaten zu defektieren (vgl. Chamberlain 1974; Stokke 2004).

Die Literatur zu Allmendegütern, maßgeblich entwickelt und untersucht durch die Arbeit der Nobelpreisgewinnerin Elinor Ostrom (vgl. z.B. 1990; 2003; Keohane/Ostrom 1995), zeigt jedoch auch die Möglichkeit auf, dass dieser Mechanismus nicht zwingenderweise zutrifft: Das Fischfangregime zum Beispiel wird im Allgemeinen als erfolgreich und effektiv eingestuft. Um genauer zu sein ist aufgrund des Rivalitätsprinzips von common pool-Ressourcen anzunehmen, dass die Anreizstrukturen der einzelnen Akteure und ihre Bereitschaft zur Kooperation positiv beeinflusst werden, denn die Bereitstellung, der Schutz und die Umverteilung dieser Güter folgen dann den Prinzipien von relative gains-Modellen (Snidal 1991a; 1991b; Keohane/ Ostrom 1995: 13) - und somit kann die Kooperationswahrscheinlichkeit mit steigender Gruppengröße wachsen. Relative Gewinne entstehen in der Regel aufgrund von Nullsummenproblematiken, bei denen die Akteure ihren Nutzen vergleichen und in Relation zu einem anderen Akteur maximieren wollen: Ein »Kuchen« wird aufgeteilt, wobei des einen Gewinn gleichzeitig den Verlust eines anderen bildet (Snidal 1991a: 387). ${ }^{11}$ Da das Rivalitätsprinzip bei öffentlichen Gütern und Externalitäten nicht zutrifft, beschränkt sich diese Nullsummenproblematik nur auf Allmendegüter.

Aufgrund dieser »Spielsituation« von Allmendegütern wird nun bei dem Konsum des Gutes durch Akteur A der Nutzen der anderen Staaten in einer Gruppe gemindert, während der Nutzen von A per definitionem gesteigert wird; daraus folgt, dass am Ende alle Akteure versuchen werden, so viel Fisch wie möglich zu fangen. Snidal (1991a: 388) zeigt, dass dies für sehr kleine Gruppen in der Tat zutrifft. Ein einzelner Staat hat hier keinen Anreiz, sein Verhalten einzuschränken, wenn nur eine kleine Anzahl an Akteuren, die dieselbe Nutzenfunktion haben, existiert. Die Staaten fahren dann z.B. mit ihren Fischereibemühungen ungehindert fort und das Allmendegut wird letztendlich zerstört.

Aufbauend auf diesem small-N-Fall zeigt Snidal jedoch (1991a: 394, 1991b: 714; vgl. Grieco 1988: 506; Ostrom 2003), dass die Problematik relativer Gewinne sich nicht auf größere Gruppen übertragen lässt. Letztlich steigt die Wahrscheinlichkeit für einen kooperativen Erfolg mit der Größe einer Gruppe für Allmendegüter. Dieser Mechanismus funktioniert wie folgt: Ähnlich der Situation eines »Versicherungsspiels« (Runge 1984) haben die Akteure einer Gruppe grundsätzlich das Interesse zu kooperieren und ein Allmendegut zu erhalten, da hier der Langzeitnutzen per se größer ist (Sen 1967). Wie bereits betont, ist Kooperation a priori aber aufgrund des Rivalitätsprinzips schwierig und in dem Fall einer nominal kleinen Gruppe wird sich das Versicherungsspiel substantiell in ein Gefangenendilemma entwickeln: alle Akteure defektieren (Snidal 1991a: 399; 1991b: 704). Aufgrund der Tatsache, dass unilaterale Kooperation in diesem Spiel das schlechteste Ergebnis darstellt, wird man

11 Diese Logik trifft auf das Fischereiregime zu: Je mehr Fisch durch einen Staat gefangen wird, desto weniger Fisch ist für die anderen Akteure verfügbar. 
selbst ebenfalls nicht kooperieren und nicht versuchen, das Allmendegut zu beschützen. Liegt nun aber eine nominal große Gruppe vor, so argumentiert Snidal (1991a: 399), dass diese Überlegungen zunehmend in den Hintergrund treten und der entscheidende Faktor die erwartete Anzahl von kooperationswilligen und defektierenden Akteuren ist. Erwartet A etwa, dass die Anzahl kooperationswilliger Staaten hinreichend groß ist, so wird A kooperieren und andere Akteure folgen ihm, da der Nutzen für den Erhalt eines Allmendeguts generell höher als für dessen Vernichtung ist. Somit formen A und andere innerhalb der gesamten Akteursgruppe eine »Untergruppe«, der es eher gelingen wird, den Langzeitnutzen durch einen höheren Anteil an dem Allmendegut gegenüber anderen Akteuren sicherzustellen. Und die Wahrscheinlichkeit, dass eine ausreichend große »Untergruppe« mit diesem Interesse besteht, steigt per definitionem mit der nominalen Größe einer Gruppe. Dies führt letztendlich gemäß des Folk-Theorems zu einem »sich selbst erzeugenden Kooperationsgleichgewicht«, das im Anschluss den erfolgreichen Schutz eines Allmendegutes sichert. Konsequenterweise argumentieren wir, dass der Schutz einer common pool-Ressource mit einer steigenden Anzahl an Akteuren in einer Gruppe wahrscheinlicher wird:

H3: Je größer eine Gruppe ist, die ein Allmendegut beschützt, desto größer ist die Wahrscheinlichkeit, dass ihr dies erfolgreich gelingt.

\section{Forschungsdesign}

\subsection{Daten}

Die Daten für die Analyse unserer Hypothesen sind der International Regimes Database (IRD) (Breitmeier et al. 1996; 2006: 39; Young/Zürn 2006) entnommen. Die Informationen dieser Datenbank sind nach Regimen, Komponenten innerhalb dieser Regime und Problemen kollektiven Handelns dieser Komponenten gegliedert. Zusammengefasst sind 23 Regime im Datensatz enthalten, wobei diese 23 Regime insgesamt 92 Regimekomponenten und 124 Probleme kollektiven Handelns aufweisen. Die Internationale Walkommission zum Beispiel hat zwei Komponenten. Eine Komponente bezieht sich auf das Walregime im Zeitraum 1946-1982, die andere auf den Zeitraum 1982-1998. Innerhalb dieser beiden Komponenten sind nun jeweils zwei Probleme kollektiven Handelns identifiziert: Zum einen Kooperationsdilemmata hinsichtlich des Bewahrens des Walbestandes und zum anderen Koordinationsprobleme bezüglich der regelkonformen Entwicklung einer Walindustrie (des Handels mit Walprodukten).

Eine Beobachtung in unserer Analyse ist demzufolge ein bestimmtes Problem kollektiven Handelns mit dem sich ein Regime befasst. Von einem analytischen Standpunkt umgeht dies nicht nur das Problem des Aggregierens von unterschiedlichen, teils sich widersprechenden Regimezielen in eine Beobachtung, sondern erhöht auch die Anzahl der beobachteten Fälle, was die Generalisierung unserer Hypothesen erhöht (vgl. Bernauer 1995: 367; King et al. 1994). Ein Nachteil dieser Methode besteht darin, dass die unterschiedlichen Probleme kollektiven Handelns innerhalb eines be- 
stimmten Regimes sehr wahrscheinlich bestimmte Elemente gemeinsam haben, so dass der Fehlerterm unseres Regressionsmodells nicht unabhängig ist. Aus diesem Grund verwenden wir Standardfehler, die für diese Heteroskedastizität des Fehlerterms über die übergeordneten Regime hinweg kontrollieren.

Die Variablen der IRD stellen im Wesentlichen keine Probleme hinsichtlich der Validität dar. Die Daten wurden durch die unabhängige Bewertung mehrerer Expertinnen und Experten des Umweltbereichs gewonnen (vgl. Breitmeier et al. 1996). Alles in allem wurden 48 Experten interviewt, mit einer Varianz von einem bis vier Befragten für jede Variable. Eine derartige Datengewinnung schließt jedoch nicht die Möglichkeit eines kognitiven Bias der Kodierer aus. Das Team der IRD begegnete diesem Problem, indem es sich nur auf Befragte verließ, die für ihre Expertise innerhalb des Feldes bekannt sind und indem mit den Kodierern über ihre Entscheidungen ausführlich diskutiert wurde (Breitmeier et al. 2006: 59). Obwohl diese Methode eine Vielzahl an Vorteilen bietet, wie z.B. die Verbindung von Fallstudien mit multivariatem, quantitativem Design, besteht jedoch auch die Möglichkeit, dass die Reliabilität zwischen den Kodierern verletzt sein könnte (Dorussen et al. 2005: 317). Um dies zu kontrollieren, betonen Breitmeier et al. (2006: 60), dass die Kodierer nur geantwortet haben, wenn sie ein hohes Vertrauen hinsichtlich der Richtigkeit ihrer Antwort hatten. Darüber hinaus berechnen wir jedoch auch Cohens (1960) $\kappa$ für die Interkoderreliabilität. Tabelle 1 zeigt Cohens $\kappa$-Werte. Alle von uns genutzten Variablen haben Interkoderreliabilitätswerte, die signifikant über den erwarteten Werten liegen. ${ }^{12}$ In Bezug auf Gruppengröße und die Gütertypen haben wir sogar volle Übereinstimmung unter den Kodierern. Die $\kappa$-Werte für Kooperationstiefe, Unsicherheit und Hegemon befinden sich innerhalb des Intervalls $[0,2 ; 0,4]$. Obwohl diese Werte sicherlich keine perfekten Übereinstimmungen haben, können sie immerhin noch als »Zufriedenstellende Werte« angesehen werden (Landis/Koch 1977).

\section{Tabelle 1: Interkoderreliabilitätswerte nach Cohens $\kappa$}

$\begin{array}{lccc} & & & \text { Erwartete } \\ \text { Kooperationstiefe } & \kappa & \text { Übereinstimmung } & \text { Übereinstimmung } \\ \text { Zahl der Verursacher (Größe) } & 0,37 & 0,54 & 0,27 \\ \text { Offentliches Gut } & 1,00 & 1,00 & 0,20 \\ \text { Allmendegüter } & 1,00 & 1,00 & 0,51 \\ \text { Externalität } & 1,00 & 1,00 & 0,52 \\ \text { Unsicherheit } & 1,00 & 1,00 & 0,72 \\ \text { Hegemon } & 0,20 & 0,45 & 0,31 \\ & 0,22 & 0,41 & 0,24\end{array}$

Anmerkung: alle Tabellenwerte signifikant unter $1 \%$.

Um den letzten, verbleibenden Bias innerhalb der Daten zu bearbeiten, wenden wir folgende Strategie an: Wir nehmen (1) die Antworten von allen Kodierern und be-

12 Die Operationalisierung der hier aufgeführten Variablen wird im nächsten Abschnitt und im Anhang detailliert beschrieben. 
rechnen darauf aufbauend (2) den Mittelwert für jede einzelne Beobachtung des Datensatzes. Für den Fall, dass die Antwort nur eines Experten verfügbar ist, nutzen wir diesen Wert ohne weitere Änderungen. Indem wir uns nun größtenteils auf Variablen verlassen, die objektiv zu erfassen sind, im Gegensatz zu items, die ein hohes Maß an Unsicherheit aufweisen, gewinnen wir abschließend zusätzliche Reliabilität und Robustheit für unsere empirischen Ergebnisse.

\subsection{Operationalisierung}

Gemäß der vorherigen Diskussion soll die abhängige Variable unserer Studie die Effektivität eines Regimes messen. Die beschriebenen Definitionen von Regimeeffektivität haben jedoch zwei fundamentale Probleme. Erstens, erweist es sich sowohl unter theoretischen als auch unter empirischen Gesichtspunkten als vergleichsweise schwierig, exakte Kriterien für die Verbesserung oder Verschlechterung eines Umweltproblems zu finden (Ward 2006; vgl. Young 1999). Zweitens, stellt sich die Frage, wie es empirisch gerechtfertigt werden kann, dass ein Regime kausal für die Veränderung eines Zustandes verantwortlich ist (Helm/Sprinz 2000: 623; Mitchell 2003: 445; Ward et al. 2004: 160).

Positiv hinsichtlich der Diskussion von Regimeeffiktiviät ist jedoch das von Downs et al. (1996) entwickelten Konzept der »Kooperationstiefe« hervorzuheben. Die Kooperationstiefe ist definiert als »the extent to which a regime demands actual behavioral changes from its participants, being measured by the density and specificity of a regime's primary rule system« (Downs et al. 1996: 383; Downs 2000: 32). Mit anderen Worten verständigen sich dabei Staaten auf ein bestimmtes Level an Kooperation in Bezug auf ein spezielles bi- oder multilaterales Problem, indem Verhaltensänderungen von Akteuren durch die gemeinsame Koordination von politischen Entscheidungen erzielt werden sollen (Keohane 1984: 15). Ein hohes Maß an Kooperationstiefe erfordert daher zum Beispiel umfassende Änderungen und Anpassungen von Staaten auf der politischen Ebene. Diese Änderungen und Anpassungen sind vertraglich in dem Regel- und Normsystem eines Regimes festgehalten. Auf der anderen Seite haben Regime einen niedrigen Level an Kooperationstiefe, wenn die Akteure planen, ihre jeweilige Umweltpolitik nur marginal zu ändern. ${ }^{13}$

13 In Bezug auf niedrige Kooperationstiefe verlangte das ursprüngliche Vertragswerk des LRTAP von seinen Mitgliedern lediglich, dass $\mathrm{SO}_{2}$-Emissionen um $30 \%$ gesenkt werden müssen. Da dies jedoch für die meisten Mitglieder nicht zu erreichen gewesen wäre, hatte das LRTAP-Regime in seiner Anfangsphase wenig Einfluss auf die Verhaltensänderung der Staaten und verursachte demzufolge auch nur wenig Kosten hinsichtlich der Vertragserfüllung. Im Umkehrschluss heißt dies für das Konzept der Kooperationstiefe aber auch, dass hier nur ein niedriges Niveau von den Staaten beschlossen wurde. Obwohl das Level an staatlicher Vertragserfüllung, aufgrund der Perzeption der Länder, dies als rationale Strategie zu sehen, als hoch einzustufen ist, war der Beitrag des LRTAP-Regimes hinsichtlich der effektiven Senkung von $\mathrm{SO}_{2}$-Emissionen gering: Die Staaten hätten ohnehin 
Neben diesen offensichtlichen Vorteilen von Kooperationstiefe als abhängiger Variable in einer Studie zu dem kooperativen Erfolg von Kollektiven lassen sich auch die beiden folgenden Punkte in der Literatur finden. Zum ersten sind wir, da Kooperationstiefe direkt die Dichte und Präzision eines Regimeregelwerkes erfasst, in der Lage, die vielen unterschiedlichen erfolgreichen und erfolglosen Kooperationsbemühungen von Staaten direkt zu messen. Das Konzept der Kooperationstiefe ermöglicht uns dabei, genaue Schlüsse über die Kooperationsbemühungen von Akteursgruppen und somit über die Effektivität eines Regimes zu ziehen. Eine hohe Kooperationstiefe ist eine notwendige Bedingung, damit ein Regime ein bestimmtes Umweltproblem im positiven Sinne beeinflussen kann. Je größer der Abstand von staatlichem Verhalten zum status quo, desto größer die Wahrscheinlichkeit, dass Staaten mehr unternehmen, um ein Umweltproblem zu lösen und desto größer die Kooperationstiefe eines Regimes. Dies impliziert die Annäherung von Staaten an ein optimales Umweltverhalten, was per definitionem die Effektivität eines Regimes erhöht.

Zweitens beeinflusst, wie oben angemerkt, die Problemstruktur nicht nur die Effektivität eines Regimes, sondern auch sein Design. Die Kooperationstiefe liegt von einem konzeptionellen Standpunkt näher an dem Design eines Regimes, als alternative Operationalisierungen von Regimeeffektivität. Kooperationstiefe reflektiert die bewussten Entscheidungen von Staaten in einer bestimmten Art und Weise zu kooperieren, um ein gewisses Maß an Umweltqualität zu erreichen. Damit sind wir auch in der Lage, das Problem intervenierender Variablen zu umgehen.

Wir sind daher der Überzeugung, dass die Kooperationstiefe eines Regimes als ein objektives Maß für den wirklichen Grad an kooperativem Erfolg zwischen Akteuren genutzt werden kann. Die IRD bietet hierzu die Variable »REGIME_SHALLOW «, welche Werte zwischen 1 (sehr geringe Kooperationstiefe) und 5 (sehr hohe Kooperationstiefe) annimmt. Tabelle 2 zeigt die Verteilung dieses items. Sehr niedrige Level an Kooperationstiefe sind durch eine sehr begrenzte Anzahl an Regeln charakterisiert und/oder Verfahrensprozeduren sind eher unterentwickelt im Vergleich zu der Spezifität von Regeln, die notwendig sind, um ein Umweltproblem zu lösen. Die Wiener Konvention oder das Klimaregime (UNFCCC) sind Institutionen solchen Typs. Zweitens, 17,74\% unserer Fälle weisen eine eher geringe Kooperationstiefe auf. Ein Beispiel: Obwohl das allgemeine Rahmenwerk der LRTAP Konvention durch ein sehr geringes Ausmaß an Kooperationstiefe gekennzeichnet ist, haben sich die Staaten entschieden, ihr Verhalten den Rahmenbedingungen der Umwelt anzupassen und ein höheres Ausmaß an Kooperation in dem Folgeprotokoll zu Schwefelemissionen zu verankern (Perrin/Bernauer 2010). Die größte Kategorie unserer Beobachtungen, d.h.

30\% ihrer Emissionen gesenkt (Victor 2006: 98). Auf der anderen Seite führten die Anhänge und Änderungen des Montreal-Protokolls in London (1990) und Kopenhagen (1992) zu einem sehr hohen Maß an Kooperationstiefe, da sich die Regimemitglieder hier auf ein starkes, bindendes, umfassendes und detailliertes Regelwerk festlegten (Breitmeier et al. 1996: 85; Oberthür 1997). Aufgrund dieser höheren Kooperationstiefe konnte das CFC-Protokoll daher erwartungsgemäß einen effektiveren Beitrag zur Lösung seines Umweltproblems beitragen, während die Mitgliedstaaten stärkere Anreize hatten, sich an die Vereinbarungen aufgrund der strengen Überwachungsmechanismen zu halten. 
41,13\%, hat für Kooperationstiefe mittlere Werte. Der Vertrag für die Bewahrung von Flora und Fauna in der Antarktis ist ein Beispiel für diese Art von Kooperationstiefe. 25,81\% unserer Fälle weisen eine eher hohe Kooperationstiefe auf, wie etwa das Walregime nach 1982. Schließlich ist ein sehr hohes Maß an Kooperationstiefe in Regimen zu finden, die ein umfassendes Regelwerk und Verhaltensprozeduren aufweisen, die relativ stark im Vergleich zu der Spezifität von Regeln, die ein Umweltproblem effektiv lösen können, sind (vgl. Breitmeier et al. 1996: 85). Das wohl bekannteste Beispiel dieser Kategorie (3,23\% unserer Fälle) ist CITES (nach 1989). Zusammengefasst betont Tabelle 2, dass weniger als 30\% unserer Fälle eine wirklich hohe Kooperationstiefe aufweisen, die jenseits der Durchschnittswerte liegt. Angesichts der ordinalen Struktur der abhängigen Variable verwenden wir geordnete logistische Regressionsmodelle (vgl. Long/Freeze 2003), um unsere Hypothesen zu testen.

Tabelle 2: Verteilung von Kooperationstiefe

Abs. Häufigkeiten

Abs. Prozentwerte

\begin{tabular}{lrr}
\hline Sehr niedrig & 15 & 12,10 \\
Niedrig & 22 & 17,74 \\
Mittel & 51 & 41,13 \\
Groß & 32 & 25,81 \\
Sehr groß & 4 & 3,23 \\
\hline Summe & 124 & 100,00
\end{tabular}

Um die Gruppengröße eines Regimes zu messen, fokussieren wir uns auf die Anzahl der Akteure, die potenziell für das Umweltproblem eines Regimes relevant sind. Diese Operationalisierung hat im Gegensatz zur wirklichen Mitgliederanzahl eines Regimes, den Vorteil, dass sie ein exogener Faktor zu Regimedesign ist (Koremenos et al. 2001: 777; vgl. Oye 1985: 21). Wir benutzen die ordinalskalierte Variable »NUMBER_CAUSERS«, die Informationen zu »how many nations were regarded as being important because of their role in causing the problem « hat (Breitmeier et al. 1996: 14). Je größer der Wert dieser Variable, desto größer die Anzahl an Verursacherstaaten, wobei der niedrigste Wert (1) für 1-5 Verursacher steht und der höchste Wert (6) für mehr als 120 Staaten.

Basierend auf unserer theoretischen Argumentation erstellen wir mit dem item »GOOD_TYPE«(Breitmeier et al. 1996: 24) drei dichotome Variablen für öffentliche Umweltgüter, Externalitäten und Allmendegüter. Um existierende Interaktionseffekte und somit den Einfluss der Gütertypen konditional abhängig von der Größe einer Gruppe zu erfassen, multiplizieren wir unsere Gruppengrößevariable mit jeder dieser Variablen. Öffentliche Umweltgüter werden in dem empirischen Regressionsmodell als Referenzkategorie verwendet und somit ausgeschlossen. 


\subsection{Kontrollvariablen}

Während die vorherigen unabhängigen Variablen zur Überprüfung der Haupthypothesen unserer Theorie herangezogen werden, müssen wir auch eine Reihe anderer Faktoren, die einen Einfluss auf den Kooperationserfolg von Regimen haben könnten, kontrollieren. Aufgrund unseres geringen Stichprobenumfangs beschränken wir uns lediglich auf die zwei prominentesten Variablen. Erstens betont Mitchell (2006: 81) den Einfluss von Unsicherheit als Teil der Problemstruktur eines Regimes. Unsicherheit bedingt, dass Akteure teure und tiefgreifende Kooperationslösungen nur zögerlich verfolgen. Auf der anderen Seite induzieren hohe Levels an Transparenz Verlässlichkeit hinsichtlich der Vertragserfüllung anderer Akteure und senken Transaktionskosten, während Staaten sich neues Wissen aneignen und Normen umsetzen können, und sich mit den Verpflichtungen eines Regimes sozialisieren (Hawkins et al. 2006; Keohane 1984: 92; Zangl 2003: 126). Eine fehlende Berücksichtigung von Unsicherheit würde zu inkonsistenten und verzerrten Ergebnissen in unseren empirischen Modellen führen. Basierend auf der Variable »PROBLEM_UNDERSTAND« der IRD (Breitmeier et al. 1996: 32) berücksichtigen wir die ordinalskalierte Variable Unsicherheit, die Werte zwischen 1 und 4 annimt. ${ }^{14}$

Barry (1978: 37) unterstreicht den Einfluss von möglichen Führerstaaten oder Hegemonien. Hegemonien in Regimen sind Akteure, die diese Institutionen organisieren und sicherstellen, dass andere Mitglieder das gemeinsame Interesse verfolgen. Durch side payments an Akteure, die dem Interesse des Hegemons nicht wohlwollend gegenüberstehen, überzeugen sie diese Staaten, zu Politiken zuzustimmen, die diese andernfalls schlechter stellen würden als im status quo (Frohlich/Oppenheimer 1970: 119; Grundig/Ward 2007). Mit anderen Worten erfassen wir aufbauend auf der Theorie hegemonialer Stabilität (vgl. Russett/Sullivan 1971: 853; Gilpin 2001), ${ }^{15}$ dass der Nutzen eines Einzelnen oder einer hinreichend kleinen k-Gruppe (Hardin 1982) aus dem Kollektivgut auch so groß sein kann, dass er die Kosten der Kollektivgutproduktion übersteigt und der Einzelne/die k-Gruppe das Gut in der Folge unilateral bereitstellt - und das Kollektiv deshalb privilegiert ist. Wir berücksichtigen demzufolge in unserem Modell die Variable »POWER_SETTING_SYMMETRY«(»Were the nations involved in regime formation roughly symmetrical in terms of issue-specific power or did the process involve sharp differences in power resources? «(Breitmeier et al. 1996: 29)), deren höchster Wert (5) für einen spezifischen Hegemon innerhalb eines Regimes steht. ${ }^{16}$

14 Die genaue Wertezuweisung ist im Anhang zu finden.

15 Letzteres wurde auch durch das k-Gruppenargument qualifiziert, d.h. k kann größer als 1 sein (Snidal 1985).

16 Die genaue Wertezuweisung ist im Anhang zu finden. 


\section{Ergebnisse}

Tabelle 3 zeigt die Ergebnisse des geordneten logistischen Regressionsmodells. Der Wald $c^{2}$-Test ist hoch signifikant für alle Modellspezifikationen. Somit kann die Nullhypothese verworfen werden, die besagt, dass alle unabhängigen Variablen zusammen keinen Einfluss auf die abhängige Variable haben. Es erscheint auch wichtig, dass die Kontrollvariablen das allgemeine Modell klar verbessern, wie in einem Likelihood Ratio-Test ( $c^{2}$-Wert von 16,41; $p$-Wert $\left.=0,000\right)$ nachgewiesen.

Tabelle 3: Geordnetes logistisches Regressionsmodell für Kooperationstiefe

\begin{tabular}{lrcc} 
& $\beta$ & Std. Fehler & $P>|z|$ \\
\hline Zahl der Verursacher (Größe) & $-0,30$ & 0,25 & 0,12 \\
Allmendegüter & $-1,47$ & 1,09 & $0,09^{*}$ \\
Externalität & 1,70 & 0,94 & $0,04^{* *}$ \\
Allmende*Größe & 0,71 & 0,48 & $0,07^{*}$ \\
Externalität*Größe & $-0,79$ & 0,41 & $0,03^{* *}$ \\
Unsicherheit & $-0,54$ & 0,30 & $0,03^{* *}$ \\
Hegemon & 0,45 & 0,33 & $0,09^{*}$ \\
\hline Cut1 & $-2,53$ & 1,26 & \\
Cut2 & $-1,28$ & 1,35 & \\
Cut3 & 0,54 & 1,30 & 115 \\
Cut4 & 3,09 & 1,37 & $-154,26$ \\
\hline$N$ & & & $29,96 * * *$ \\
Log Pseudo Likelihood & & & 0,15 \\
Wald $\chi^{2}$ (7) & & & \\
Pseudo $R^{2}$ & & & \\
\hline
\end{tabular}

Anmerkungen: * signifikant auf 10\%; ** signifikant auf 5\%; *** signifikant auf $1 \%$ (einseitiger Test).

Die Kontrollvariablen sind mit der Argumentation in der bisherigen Literatur konsistent und haben die vorhergesagten Vorzeichen. Unsicherheit hat einen negativen Einfluss auf die Kooperationstiefe eines Regimes; der Einfluss ist auf dem 5\% Level signifikant. Dies bedeutet: Je größer das Maß an Unsicherheit und der Mangel an Information über ein Umweltproblem sind, desto geringer ist das Ausmaß an kooperativem Erfolg eines Regimes und somit auch der Mitgliedstaaten, die sich mit diesem Problem befassen. Das LRTAP ist ein klassisches Beispiel für diese generelle Beziehung. Zu Beginn des Regimes in den 1970ern wusste man über die Konsequenzen von grenzüberschreitender Luftverschmutzung wenig; das Regime erwies sich dementsprechend ineffektiv in der Lösung dieses Problems. Weiterhin ist die Koeffizientenschätzung für Hegemon positiv und auf dem 10\% Level signifikant. Dies zeigt, dass in der Tat eine ungleiche Verteilung an Macht und Einfluss in Bezug auf ein Kooperationsproblem dazu beitragen kann, dass Probleme kollektiven Handelns eher gelöst werden. Wenn eine Gruppe von Akteuren eine hinreichend kleine k-Gruppe im besten Falle einen spezifischen Hegemon - beinhaltet, so erhöhen sich signifikant 
die Chancen, dass kollektive Güter zur Verfügung gestellt werden. Die Inter-American Tropical Tuna Convention ist ein Paradebeispiel für diesen Fall, da das Regime trotz seiner 13 Mitglieder nicht nur stark abhängig von den USA ist, sondern auch von diesem Land dominiert wird.

Hinsichtlich unserer Kernhypothesen sei anzumerken, dass die einzelnen Terme, d.h. die beiden Gütertypenvariablen, die Gruppengrößevariable und die Multiplikationsitems, aufgrund der Interaktionseffekte nicht direkt aus Tabelle 3 interpretiert werden können (vgl. Ai/Norton 2003; Braumoeller 2004; Brambor et al. 2006). In geordneten logistischen Regressionsmodellen können auch Koeffizienten nicht als Prozentänderungen oder marginale Effekte interpretiert werden. Lediglich das Vorzeichen gibt Aufschluss über die Art des Einflusses einer Variable. Um unsere Ergebnisse besser interpretieren zu können, berechnen wir daher die vorhergesagten Wahrscheinlichkeiten für Kooperationstiefe aufgrund der verschiedenen Gütertypen abhängig von Größe. Abbildung 1 und Tabelle 4 zeigen unsere Berechnungen für große/sehr große Kooperationstiefe.

\section{Abbildung 1: Umweltgüter in Beziehung zu großer/sehr großer Kooperationstiefe}

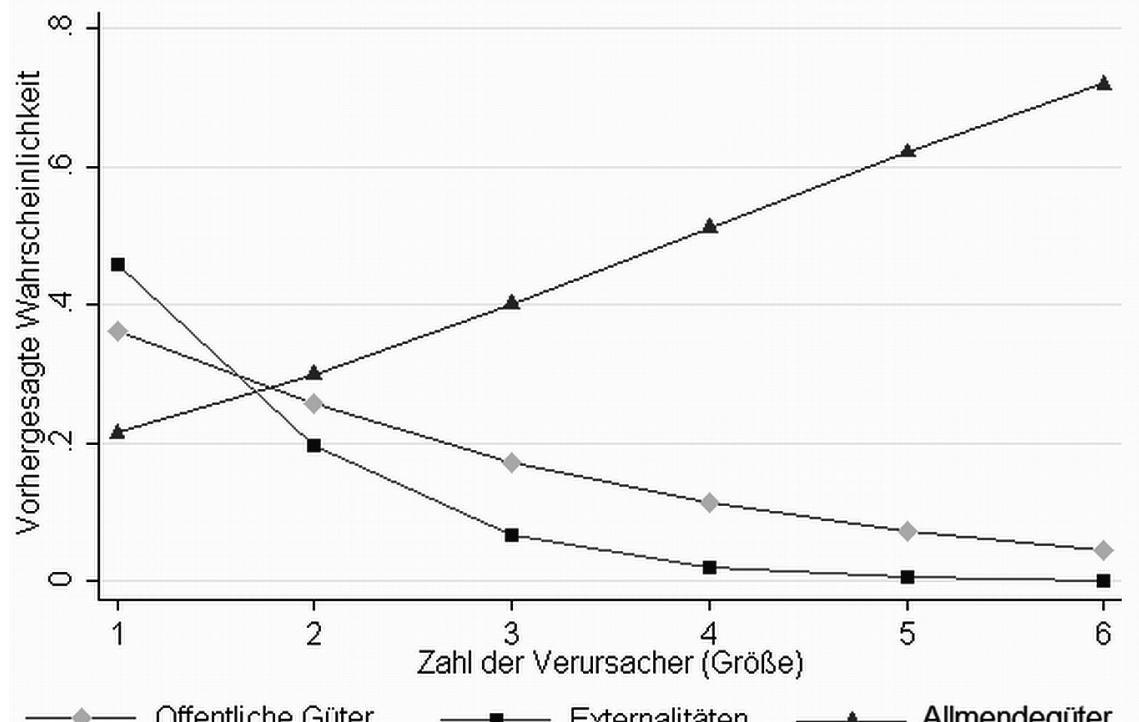

Anmerkungen: Berechnungen wurden für die einzelnen Variablen vorgenommen während alle übrigen Faktoren konstant auf dem jeweiligen Mittelwert gehalten sind. 
Tabelle 4: Vorhergesagte Wahrscheinlichkeiten für große/sehr große Kooperationstiefe nach Umweltgüter und Gruppengröße

\begin{tabular}{r|rrr} 
Zahl der Verursacher (Größe) & Öffentliche Güter & Allmendegüter & Externalitäten \\
\hline $1-5$ & 0,36 & 0,21 & 0,46 \\
$6-15$ & 0,26 & 0,30 & 0,20 \\
$16-30$ & 0,17 & 0,40 & 0,07 \\
$31-60$ & 0,11 & 0,51 & 0,02 \\
$60-120$ & 0,07 & 0,62 & 0,01 \\
Mehr als 120 & 0,04 & 0,72 & 0,00
\end{tabular}

In Übereinstimmung mit unseren Hypothesen erhalten wir überzeugende Unterstützung für unsere Theorie. Komplementär zu unserer Argumentation in $H 3$ zeigen Abbildung 1 und Tabelle 4, dass die Wahrscheinlichkeit, ein großes Ausmaß an Kooperationserfolg zwischen den Akteuren zu erhalten, um ungefähr 51\%-Punkte steigt je größer die Gruppe, die ein Allmendegut erhalten möchte, ist. Allmendegüter werden somit eher in Regimen mit einer großen Zahl an Mitgliedern geschützt. CITES zum Beispiel sieht im Wesentlichen jedes Land als relevant für den Schutz von bedrohten Arten von Flora und Fauna an. Vor diesem Hintergrund versucht diese Institution, Allmendegüter zu beschützen und hat, konsistent mit unserer Argumentation und den Resultaten, eine der höchsten Kooperationstiefen aller Regime. Konsequenterweise scheint es, dass vergleichsweise kleine Gruppen an Akteuren, d.h. 16 Staaten oder weniger, es schwieriger finden, Allmendegüter effektiv zu schützen. Auf der anderen Seite zeigen Abbildung 1 und Tabelle 4, dass große Gruppen, die sich mit öffentlichen Umweltgütern oder Externalitäten beschäftigen, weniger erfolgreich sind. Wenn wir uns auf den höchsten Wert des Gruppengrößeitems zubewegen, fallen die vorhergesagten Wahrscheinlichkeiten um ca. 32\%-Punkte für öffentliche Güter (H1) und um ungefähr 46\%-Punkte für Externalitäten $(H 2)$. Die UNFCCC als Institution, die weltweit alle Staaten hinsichtlich des Klimaschutzes (globales öffentliches Gut) adressiert, betont diese Beziehung. Dieses Regime weist in unseren Daten eine der niedrigsten Kooperationstiefen auf. Für Externalitätsprobleme bedeutet dies, dass große Kooperationstiefe signifikant weniger wahrscheinlich für solche Gütertypen gegeben ist, wenn eine große Gruppe an Akteuren involviert ist. Das LRTAP Regime als wohl bekannteste Institution zur Bewältigung von Umweltexternalitäten stellt einen Fall dar, der diese Beziehung widerspiegelt. Unsere Resultate zeigen somit, dass kleine Gruppen eher fähig sind zu kooperieren und sich auf präzise Regelsysteme zu einigen, wenn das Hauptanliegen die Beseitigung einer Externalität ist. Zusammengefasst heißt dies für öffentliche Umweltgüter und Externalitäten: Je größer die Gruppe an relevanten Akteuren ist, desto geringer ist die Wahrscheinlichkeit, dass diese letztendlich kooperativ erfolgreich sein werden und ein hohes Maß an Kooperationstiefe erreichen. Wir erhalten somit Hinweise, dass all unsere Hypothesen einen hohen Wahrheitsgehalt aufweisen. 
Abbildung 2: Umweltgüter in Beziehung zu niedriger/sehr niedriger Kooperationstiefe

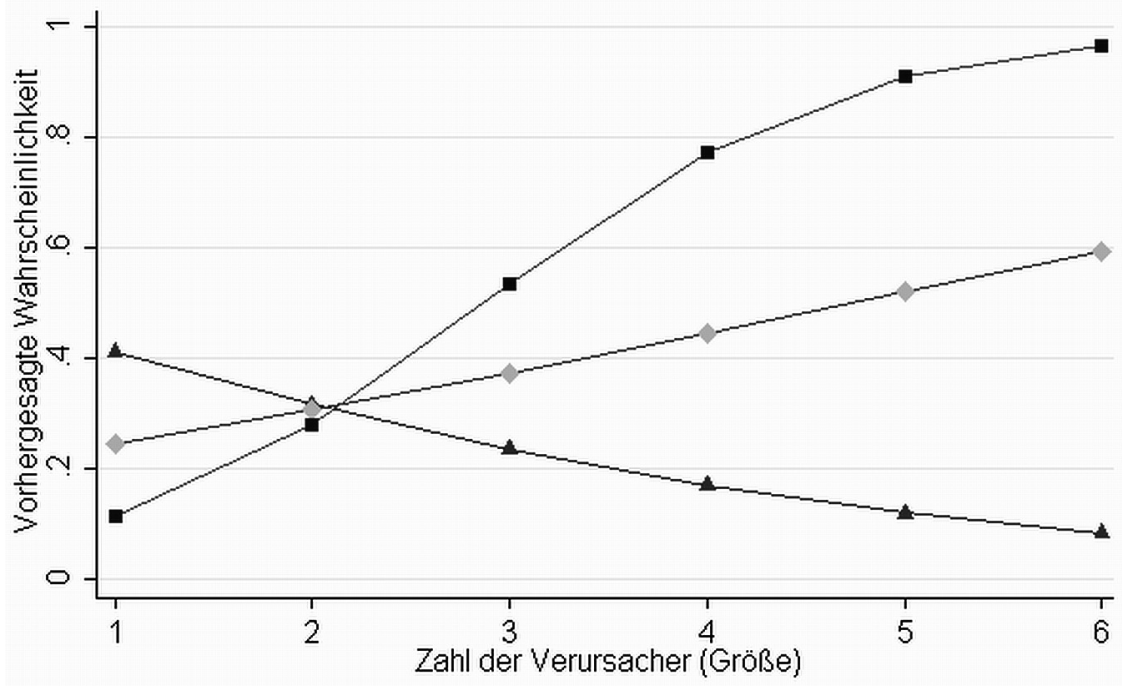

— Offentliche Güter

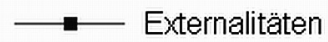

Allmendegüter

Anmerkungen: Berechnungen wurden für die einzelnen Variablen vorgenommen während alle übrigen Faktoren konstant auf dem jeweiligen Mittelwert gehalten sind.

Tabelle 5: Vorhergesagte Wahrscheinlichkeiten für niedrige/sehr niedrige Kooperationstiefe nach Umweltgüter und Gruppengröße

\begin{tabular}{r|rrr} 
Zahl der Verursacher (Größe) & Öffentliche Güter & Allmendegüter & Externalitäten \\
\hline $1-5$ & 0,25 & 0,41 & 0,12 \\
$6-15$ & 0,31 & 0,32 & 0,28 \\
$16-30$ & 0,37 & 0,24 & 0,54 \\
$31-60$ & 0,45 & 0,17 & 0,77 \\
$60-120$ & 0,52 & 0,12 & 0,91 \\
Mehr als 120 & 0,59 & 0,08 & 0,97
\end{tabular}

Um die Robustheit unserer Resultate zu sichern, überprüfen wir unsere Hypothesen auch für die vorhergesagten Wahrscheinlichkeiten von Regimen mit niedriger/sehr niedriger Kooperationstiefe. Die Berechnungen sind in Abbildung 2 und Tabelle 5 aufgezeigt und bestätigen die Ergebnisse unserer bisherigen Analysen. Die vorhergesagten Wahrscheinlichkeiten von Gruppen mit niedriger Kooperationstiefe sinken um ca. 32\%-Punkte für Allmendegüter, wenn wir uns von kleinen Gruppen zu Akteurskonstellationen von mehr als 120 Verursachern bewegen. Dies ist konsistent mit 
H3. In Übereinstimmung mit unserer Theorie steigen auch die vorhergesagten Wahrscheinlichkeiten für öffentliche Umweltgüter oder Externalitäten in Abbildung 2 und Tabelle 5. Im Detail sehen wir eine Steigung von ca. 35\%-Punkten für öffentliche Güter $(H 1)$ und ca. 85\%-Punkten für Umweltexternalitäten $(H 2)$, um ein niedriges/ sehr niedriges Level an Kooperationstiefe zu erreichen, wenn wir uns dem Maximum von Größe approximativ nähern. Mit anderen Worten: Je größer die Gruppen an relevanten Staatsakteuren sind, desto weniger effektiv sind Regime, die mit öffentlichen Umweltgütern oder Externalitäten handeln.

\section{Schlussfolgerungen}

In dieser Abhandlung haben wir versucht, unser Verständnis und unser Wissen über Probleme kollektiven Handelns zu erweitern, indem existierende theoretische Argumente zu Gruppengröße und kollektiven Gütern (Olson 1965) im Rahmen von internationalen Umweltregimen empirisch getestet wurden. In diesem Zusammenhang entwickelten wir unter Berücksichtigung bestehender Literatur ein theoretisches Modell, das den Effekt von Gruppengröße auf die Kooperationstiefe von Staaten, abhängig von öffentlichen Gütern, Externalitäten und Allmendegütern untersucht.

In unserer Analyse fanden wir eine Reihe von Ergebnissen, die darauf hindeuten, dass unsere Hypothesen richtig sind. Größere Gruppen scheinen in der Tat Probleme zu haben, öffentliche Umweltgüter $(H I)$ optimal bereitzustellen und Umweltexternalitäten effektiv zu beseitigen ( $H 2)$. Weiterhin betont unsere Analyse, dass größere Gruppen wahrscheinlich weitreichender in der Lage sind, Allmendegüter zu schützen (H3). Mit anderen Worten: Indem wir auf die Berechnungen des geordneten logistischen Regressionsmodells zurückgreifen, zeigt unsere graphische Repräsentation der substantiellen Effekte von Gütertyp und Gruppengröße Unterstützung für $H 1, H 2$ und H3. Die vorhergesagten Wahrscheinlichkeiten für Kooperationstiefe deuten an, abhängig von Gruppengröße und dem spezifischen Umweltgut, dass unsere Hypothesen richtig sind. Bei großen Gruppen, die versuchen, ein öffentliches Umweltgut bereitzustellen oder eine Externalität beseitigen wollen, ist die Wahrscheinlichkeit eines Erfolges gering, da unsere Ergebnisse hier sehr niedrige Werte für Kooperationstiefe aufzeigen. Auf der anderen Seite sind Gruppen hinsichtlich ihres Kooperationserfolges effektiver, wenn sie eine common pool-Ressource beschützen wollen und dies in vergleichsweise großen Gruppen erfolgt.

Mit dieser Analyse ordnet sich diese Abhandlung in die Reihe von Forschungsarbeiten zu Problemen kollektiven Handelns im Rahmen der Regimeforschung ein. Sie ist nach unserem Wissensstand die erste quantitative Arbeit der Regimeliteratur, die sich systematisch mit den Bereitstellungssitationen von Kollektivgütern befasst. So hat unser Aufsatz nicht nur empirische Hinweise für die Validität von Olsons (1965) Argumenten gefunden, sondern auch gezeigt, dass derselbe kausale Mechanismus nicht zwingend auf Allmendegüter ausgeweitet werden kann oder aber dass das kGruppenargument - operationalisiert durch die Variable Hegemon - an Bedeutung verloren haben könnte. 
In Bezug auf die praktischen Implikationen unserer Forschung und in Hinsicht auf die Debatten wie internationale Umweltorganisationen neu gestaltet werden könnten (vgl. Najam 2003; Victor 2006: 95), zeigen unsere Ergebnisse abschließend auch, dass sich Politiker des Rahmens, in dem Entscheidungen getroffen werden, sehr genau bewusst sein sollten. Globale Umweltregime oder eine »Weltumweltorganisation« müssen nicht notwendigerweise effektiver sein, um öffentliche Umweltgüter zu schützen (vgl. Oberthür/Gehring 2004). Auf der anderen Seite und vor dem Hintergrund der Klimakonferenzen in Kopenhagen 2009 und Cancun 2010 ließe sich zunächst vermuten, dass es effektiver ist solche Probleme in einem kleineren Rahmen zu adressieren oder etwa Mitglieder aus einem Regime auszuschließen. Anders formuliert könnte es vor einem praktischen Hintergrund demnach sinnvoll erscheinen, dass etwa das Antarktisregime (öffentliches Gut) oder das LRTAP-Regime (Externalität) signifikant die Anzahl der Mitgliedsstaaten senkt, um ein größeres Ausmaß an kooperativem Erfolg erzielen zu können. Dies sind jedoch eher absurde Vorschläge, da Regimeprobleme und deren Struktur in der Regel eng an die potentielle Regimegröße gekoppelt sind (Koremenos et al. 2001). Es könnte sich demzufolge bei dem von uns behandelten Sachverhalt wohl um ein Dilemma in der Regimeforschung - als um eine Frage von choice - handeln.

\section{Literatur}

Agrawal, Arun/Goyal, Sanjeev 2003: Group Size and Collective Action: Third-Party Monitoring in Common Pool Resources, in: Comparative Political Studies 34: 1, 63-93.

Ai, Chunrong/Norton, Edward C. 2003: Interaction Terms in Logit and Probit Models, in: Economics Letters 80: 1, 123-129.

Axelrod, Robert/Keohane, Robert O. 1985: Achieving Cooperation Under Anarchy: Strategies And Institutions, in: World Politics 38: 1, 226-254.

Barkin, Samuel J./Shambaugh, George E. 1996: Common-Pool Resources And International Environmental Politics, in: Environmental Politics 5: 3, 429-447.

Barrett, Scott 1999: A Theory of Full International Cooperation, in: Journal of Theoretical Politics 11: 4, 519-541.

Barrett, Scott 2007: Why Cooperate? The Incentive to Supply Global Public Goods, Oxford.

Barry, Brian 1978: Sociologists, Economists, and Democracy, Chicago, IL.

Bernauer, Thomas 1995: The Effect of International Environmental Institutions: How We Might Learn More, in: International Organization 49: 2, 351-377.

Brambor, Thomas/Clark, William R./Golder, Matt 2006: Understanding Interaction Models: Improving Empirical Analysis, in: Political Analysis 14: 1, 63-82.

Braumoeller, Bear F. 2004: Hypothesis Testing and Multplicative Interaction Terms, in: International Organization 58: 4, 807-820.

Breitmeier, Helmut/Levy Marc A./Young, Oran R./Zürn, Michael 1996: IIASA International Regimes Database - Data Protocol (IIASA Working Paper W), Laxenburg, 96-159.

Breitmeier, Helmut/Young, Oran R./Zürn, Michael 2006: Analyzing International Environmental Regimes: From Case Study to Database, Cambridge, MA.

Chamberlain, John 1974: Provision of Collective Goods As a Function of Group Size, in: American Political Science Review 68: 2, 707-716.

Coase, Ronald H. 1960: The Problem of Social Costs, in: Journal of Law and Economics 3, 1-31. 
Cohen, Jacob 1960: A Coefficient of Agreement for Nominal Scales, in: Educational and Psychological Measurement 20: 1, 37-46.

Dixit, Avinash/Olson, Mancur 2000: Does Voluntary Participation Undermine the Coase Theorem?, in: Journal of Public Economics 76: 3, 309-335.

Dorussen, Han/Lenz, Hartmut/Blavoukos, Spyros 2005: Assessing the Reliability and Validity of Expert Interviews, in: European Union Politics 6: 3, 315-337.

Downs, George W. 2000: Constructing Effective Environmental Regimes, in: Annual Review of Political Science 3, 25-42.

Downs, George W./Rocke, David M./Barsoom, Peter N. 1996: Is The Good News about Compliance Good News about Cooperation?, in: International Organization 50: 3, 379-406.

Fearon, James D. 1998: Bargaining, Enforcement and International Cooperation, in: International Organization 52: 2, 269-305.

Frohlich, Norman/Oppenheimer, Joe A. 1970: I get by with a Little Help from my Friends, in: World Politics 23: 1, 104-120.

Gilpin, Ronald 2001: Global Political Economy: Understanding the International Economic Order, Princeton, NJ.

Grieco, Joseph M. 1988: Anarchy and the Limits of Cooperation: A Realist Critique of the Newest Liberal Institutionalism, in: International Organization 42: 3, 485-507.

Grundig, Frank/Ward, Hugh 2007: Hegemonic Leadership or Leadership Competition? Beyond Hegemonic Stability Theory, unv. Ms.

Hardin, Garrett 1970: Die Tragik der Allmende, in: Lohmann, Michael (Hrsg.): Gefährdete Zukunft - Prognosen angloamerikanischer Wissenschaftler, München, 30-48.

Hardin, Russell 1982: Collective Action, Baltimore, MD.

Harris, Paul 2007: Collective Action on Climate Change: The Logic of Regime Failure, in: Natural Resources Journal 47: 1, 195-224.

Hasenclever, Andreas/Mayer, Peter/Rittberger, Volker 1997: Theories of International Regimes, Cambridge.

Hawkins, Darren G./Lake, David A./Nielson, Daniel L./Tierney, Michael 2006: Delegation under Anarchy: States, International Organizations, and Principal-Agent Theory, in: Hawkins, Darren G./Lake, David A./Nielson, Daniel L./Tierney, Michael (Hrsg.): Delegation and Agency in International Organizations, Cambridge, 3-38.

Helm, Carsten/Sprinz, Detlef 2000: Measuring the Effectiveness of International Environmental Regimes, in: Journal of Conflict Resolution 44: 5, 630-652.

Holzinger, Katharina 2001: Aggregation Technology of Common Goods and its Strategic Consequences: Global Warming, Biodiversity, and Siting Conflicts, in: European Journal of Political Research 40: 2, 117-138.

Hovi, Jon/Sprinz, Detlef/Underdal, Arild 2003: The Oslo-Potsdam Solution to Measuring Regime Effectiveness: Critique, Response, and the Road Ahead, in: Global Environmental Politics 3: 3, 74-96.

Isaac, Mark R./Walker, James M./Williams, Arlington W. 1994: Group Size and the Voluntary Provision of Public Goods - Experimental Evidence Utilizing Large Groups, in: Journal of Public Economics 54: 1, 1-36.

Kaul, Inge/Conceicao, Pedro/Goulven, Katell Le/Mendoza, Ronald U. 2003: Providing Public Goods - Managing Globalization, Oxford.

Keohane, Robert O. 1984: After Hegemony: Cooperation and Discord in the World Political Economy, Princeton, NJ.

Keohane, Robert O./Ostrom, Elinor (Hrsg.) 1995: Local Commons and Global Interdependence: Heterogeneity and Cooperation in Two Domains, London.

King, Gary/Keohane, Robert O./Verba, Sidney 1994: Designing Social Inquiry: Scientific Inference in Qualitative Research, Princeton, NJ.

Koremenos, Barbara/Lipson, Charles/Snidal, Duncan 2001: The Rational Design of International Institutions, in: International Organization 55: 4, 761- 799. 
Krasner, Stephen 1983: Structural Causes and Regime Consequences: Regimes as Intervening Variables, in: Krasner, Stephen (Hrsg.): International Regimes, Cornell, NY, 1-22.

Kydd, Andrew/Snidal, Duncan 1993: Progress in Game-theoretical Analysis of International Regimes, in: Rittberger, Volker/Mayer, Peter (Hrsg.): Regime Theory and International Relations, Oxford, 112-135.

Landis, Richard J./Koch, Gary G. 1977: The Measurement of Observer Agreement for Categorical Data, in: Biometrics 33: 1, 159-174.

Long, J. Scott/Freeze, Jeremy 2003: Regression Models for Categorical Dependent Variables Using Stata, College Station, TX.

Martin, Lisa L. 1992: Interests, Power, and Multilateralism, in: International Organization 46: 4, 765-792.

Martin, Lisa L. 1995: Heterogeneity, Linkage and Common Problems, in: Keohane, Robert O./ Ostrom, Elinor (Hrsg.): Local Commons and Global Interdependence: Heterogeneity and Cooperation in Two Domains. London: SAGE Publications, 71-91.

Miles, Edward L./Underdal, Arild/Andresen, Steinar/Wettestad, Jørgen/Skaerseth, Jon B./Carlin, Elaine M. (Hrsg.) 2002: Environmental Regime Effectiveness, Cambridge, MA.

Mitchell, Ronald B. 1996: Compliance Theory: An Overview, in: Cameron, James/Werksman, Jacob/Roderick, Peter (Hrsg.): Improving Compliance with International Environmental Law, London, 3-28.

Mitchell, Ronald B. 2002: Of Course International Institutions Matter: But When and How?, in: Biermann, Frank/Brohm, Rainier/Dingwerth, Klaus (Hrsg.): Proceedings of the 2001 Berlin Conference on the Human Dimensions of Global Environmental Change: Global Environmental Change and the Nation State (PIK Report No. 80), Potsdam, 16-25.

Mitchell, Ronald B. 2003: International Environmental Agreements: A Survey of Their Features, Formation, and Effects, in: Annual Review of Environment and Resources 28: 3, 429-461.

Mitchell, Ronald B. 2006: Problem Structure, Institutional Design, and the Relative Effectiveness of International Environmental Agreements, in: Global Environmental Politics 6: 3, 72-89.

Mueller, Dennis C. 2003: Public Choice III, Cambridge.

Najam, Adil 2003: The Case against a New International Environmental Organization, in: Global Governance 9: 3, 367-384.

North, Douglass 1990: Institutions, Institutional Change and Economic Performance, Cambridge.

Oberthür, Sebastian 1997: Umweltschutz durch internationale Regime: Interessen, Verhandlungsprozesse, Wirkungen, Opladen.

Oberthür, Sebastian/Gehring, Thomas 2004: Reforming International Environmental Governance: An Institutional Critique of the Proposal for a World Environment Organization, in: International Environmental Agreements: Politics, Law and Economics 4: 4, 359-381.

Olson, Mancur 1965: The Logic of Collective Action: Public Goods and the Theory of Groups, Cambridge, MA.

Olson, Mancur/Zeckhauser, Richard 1966: An Economic Theory of Alliances, in: The Review of Economics and Statistics 48: 3, 266-279.

Ostrom, Elinor 1990: Governing the Commons: The Evolution of Institutions for Collective Action, Cambridge.

Ostrom, Elinor 2003: How Types of Goods and Property Rights Jointly Affect Collective Action, in: Journal of Theoretical Politics 15: 3, 239-270.

Oye, Kenneth 1985: Explaining Cooperation under Anarchy: Hypotheses and Strategies, in: World Politics 38: 1, 1-24.

Perrin, Sophie/Bernauer, Thomas 2010: International Regime Formation Revisited: Explaining Ratification Behaviour with Respect to Long-Range Transboundary Air Pollution Agreements in Europe, in: European Union Politics 11: 3, 405-426. 
Poteete, Amy R./Ostrom, Elinor 2008: Fifteen Years of Empirical Research on Collective Action in Natural Resource Management: Struggling to Build Large-N Databases Based on Qualitative Research, in: World Development 36: 1, 176-195.

Ringquist, Evan J./Kostadinova, Tatiana 2005: Assessing the Effectiveness of International Environmental Agreements: The Case of the 1985 Helsinki Protocol, in: American Journal of Political Science 49: 1, 86-102.

Rittberger, Volker/Zürn, Michael 1990: Towards Regulated Anarchy in East-West Relations, in: Rittberger, Volker (Hrsg.): International Regimes in East-West Politics, London, 9-63.

Runge, Carlisle F. 1984: Institutions and the Free Rider: The Assurance Problem in Collective Action, in: Journal of Politics 46: 1, 154-181.

Russett, Bruce M./Sullivan, John D. 1971: Collective Goods and International Organizations, in: International Organization 25: 4, 845-865.

Sandler, Todd 1992: Collective Action: Theory and Applications, Ann Arbor, MI.

Sandler, Todd 2004: Global Collective Action, Cambridge.

Schelling, Thomas C. 1973: Hockey, Helmets, Concealed Weapons, and Daylight Saving - A Study of Binary Choices with Externalities, in: Journal of Conflict Resolution 17: 3, 381-428.

Sen, Amartya 1967: Isolation, Assurance and the Social Rate of Discount, in: Quarterly Journal of Economics 81: 1, 112-124.

Shimizu, Hirofumi/Sandler, Todd 2002: Peacekeeping and Burden-Sharing, 1994-2000, in: Journal of Peace Research 39: 6, 651-668.

Simmons, Beth A./Martin, Lisa L. 2002: International Organizations and Institutions, in: Carlesnaes, Walter/Risse, Thomas Risse/Simmons, Beth A. (Hrsg.): Handbook of International Relations, London, 192-211.

Snidal, Duncan 1985: The Limits of Hegemonic Stability Theory, in: International Organization 39: 4, 579-614.

Snidal, Duncan 1991a: International Cooperation Among Relative Gains Maximizers, in: International Studies Quarterly 35: 4, 387-402.

Snidal, Duncan 1991b: Relative Gains and the Patterns of International Cooperation, in: American Political Science Review 85: 3, 701-726.

Snidal, Duncan 1996: Political Economy and International Institutions, in: International Review of Law and Economics 16: 1, 121-137.

Stokke, Olav S. 2004: Boolean Analysis, Mechanisms, and the Study of Regime Effectiveness, in: Underdal, Arild/Young, Oran R. (Hrsg.): Regime Consequences: Methodological Challenges and Research Strategies, Dordrecht, 87-119.

Taylor, Michael 1987: The Possibility of Cooperation, Cambridge.

Underdal, Arild 2002: One Question, Two Answers, in: Miles, Edward L./Underdal, Arild/Andresen, Steinar/Wettestad, Jørgen/Skaerseth, Jon B./Carlin, Elaine M. (Hrsg.): Environmental Regime Effectiveness, Cambridge, MA, 3-45.

Victor, David G. 2006: Toward Effective International Cooperation on Climate Change: Numbers, Interests and Institutions, in: Global Environmental Politics 6: 3, 90-103.

Ward, Hugh 2006: International Linkages and Environmental Sustainability: The Effectiveness of the Regime Network, in: Journal of Peace Research 43: 2, 149-166.

Ward, Hugh/Grundig, Frank/Zorick, Ethan 2004: Formal Theory and Regime Effectiveness: Rational Players, Irrational Regimes, in: Underdal, Arild/Young, Oran R. (Hrsg.): Regime Consequences: Methodological Challenges and Research Strategies, Dordrecht, 151-183.

Weiss, Edith/Jacobson, Harold K. 1998: Engaging Countries: Strengthening Compliance with International Environmental Accords, Cambridge, MA.

Young, Oran R. 1982: Regime Dynamics: The Rise and Fall of International Regimes, in: International Organization 36: 2, 277-297.

Young, Oran R. (Hrsg.) 1999: The Effectiveness of International Environmental Regimes: Causal Connections and Behavioral Mechanisms, Cambridge, MA. 
Young, Oran R./Zürn, Michael 2006: The International Regimes Database: Designing and Using a Sophisticated Tool for Institutional Analysis, in: Global Environmental Politics 6: 3, 121-143.

Zangl, Bernhard 2003: Regimetheorie, in: Schieder, Siegfried/Spindler, Manuela (Hrsg.): Theorien der Internationalen Beziehungen, Opladen, 117-140.

Zürn, Michael 1992: Interessen und Institutionen in der Internationalen Politik. Grundlegungen und Anwendungen des situationsstrukturellen Ansatzes, Opladen.

Zürn, Michael 1998: The Rise of International Environmental Politics: A Review of Current Research, in: World Politics 50: 4, 617-649.

Anhang - Kodierungsanweisungen der Variablen der IRD (Breitmeier et al. 1996)

1. Kooperationstiefe: Is the regime shallow or deep as measured by the density and specificity of its rules?

$1=$ Very shallow: Compared to the density of rules considered necessary for managing the problems in the issue area, the regime comprises only a very limited number of rules, and/or established rules are rather weak compared to the specificity of the rules considered necessary for managing the problems in the issue area.

$2=$ Shallow: Between 1 and 3 on the scale.

3 = Medium: Compared to the density of rules considered necessary for managing the problems in the issue area, the regime comprises a sizable number of rules to manage the problem and/or established rules have developed some strength compared to the specificity of the rules considered necessary for managing the problems in the issue area.

$4=$ Deep: Between 3 and 5 on the scale.

$5=$ Very deep: Compared to the density of rules considered necessary for managing the problems in the issue area, the regime comprises a very comprehensive set of rules and/or established rules are rather strong compared to the specificity of the rules considered necessary for managing the problems in the issue area (e.g., the adjustments and amendments to the Montreal Protocol (1987) adopted in London (1990) and Copenhagen (1992) led to a rather deep regime with comprehensive and strong rules).

2. Zahl der Verursacher (Größe): How many nations were regarded as being important because of their role in causing the problem? Code each problem mentioned under PROBLEM above.

$1=1-5$

$2=6-15$

$3=16-30$

$4=31-60$

$5=60-120$

$6=$ More than 120 
3. Gütertyp: Does the problem involve supplying a collective good, regulating the use of a common pool resource, managing a shared natural resource, or controlling transboundary externalities? ${ }^{17}$

$0=$ None of the types

$1=$ Collective (or public) good

$2=$ Common pool resource

$3=$ Shared natural resource

$4=$ Common pool resource and shared

$5=$ Transboundary externalities

\section{Unsicherheit: Was the nature of the problem well understood? ${ }^{18}$}

$1=$ Very strongly established understanding: There was general consensus regarding nature, causes, and consequences of the problem, as well as regarding solutions and what should be maximized in the issue area.

$2=$ Strongly established understanding: Between 1 and 3 on the scale.

3 = Partially established understanding: Consensus was partially achieved, either by consensus on some but not all of the different variables (nature, causes, and consequences of the problem as well as solutions and what should be maximized in the issue area) or by generally growing, but still not fully developed, consensus on all of the different variables.

4 = Low established understanding: Between 3 and 5 on the scale.

$5=$ Not at all established: Understanding was not established with regard to nature, causes, and consequences of the problem, or to solutions or what should be maximized in the issue area.

POWER

5. Hegemon: Were the nations involved in regime formation roughly symmetrical in terms of issue-specific power or did the process involve sharp differences in power resources?

1 = Completely even distribution: Issue-specific power resources are evenly distributed among nations.

$2=$ Slightly uneven distribution: Besides slightly uneven distribution, no single nation has a greater ability to get other nations to do something they otherwise would not do.

17 Die Kategorien $» 0 \ll$, $» 3 \ll$ und $» 4$ « werden in unserem Forschungsdesign nicht berücksichtigt und sind daher ausgeschlossen.

18 Kategorie $» 5 \ll$ ist in unserem Datensatz für keines der Regime vorhanden. 
3 = Considerable unevenness: Uneven distribution of power resources can lead to more powerful actors being able to get other nations to do something they otherwise would not do with regard to a limited number of issues in the issue area.

4 = Highly uneven distribution: Very uneven distribution of power resources can lead to more powerful actors being able to get other nations to do something they otherwise would not do with regard to a significant number of issues in the issue area.

5 = Issue-specific hegemon present: One single actor can get all other actors to do things that they otherwise would not do with regard to nearly all issues at stake in the issue area. 